\title{
Plasma Risperidone-related Measures in Children and Adolescents with Oppositional Defiant/Conduct Disorders
}

\author{
Daria Piacentino $^{1,2}$, Georgios D. Kotzalidis ${ }^{3}$, Georgios Schoretsanitis ${ }^{4}$, Michael Paulzen ${ }^{5,6}$, Ekkehard Haen ${ }^{7}$, \\ Simone Cappelletti ${ }^{8}$, Giancarlo Giupponi ${ }^{2}$, Michael Grözinger ${ }^{6}$, Andreas Conca ${ }^{2}$ \\ ${ }^{1}$ Section on Clinical Psychoneuroendocrinology and Neuropsychopharmacology, National Institute on Alcohol Abuse and Alcoholism, Division \\ of Intramural Clinical and Basic Research and National Institute on Drug Abuse, Intramural Research Program, National Institutes of Health, \\ Bethesda, MD, USA, ${ }^{2}$ Department of Psychiatry, Central Hospital, Health Agency of South Tyrol, Bozen, Italy, ${ }^{3}$ NESMOS Department \\ (Neurosciences, Mental Health, and Sensory Organs), Sapienza University of Rome, School of Medicine and Psychology, Sant'Andrea Hospital, \\ Rome, Italy, ${ }^{4}$ Department of Psychiatry Research, The Zucker Hillside Hospital, Northwell Health, Glen Oaks, NY, USA, ${ }^{5}$ Alexianer Hospital \\ Aachen, ${ }^{6}$ Department of Psychiatry, Psychotherapy and Psychosomatics, RWTH Aachen University, Aachen, ${ }^{7}$ Clinical Pharmacology, Department \\ of Psychiatry and Psychotherapy and Department of Pharmacology and Toxicology, University of Regensburg, Regensburg, Germany, \\ ${ }^{8}$ Anatomical, Histological, Forensic Medicine, and Orthopedic Sciences (SAIMLAL) Department, Sapienza University, Rome, Italy
}

\begin{abstract}
Objective: Therapeutic drug monitoring helps clinicians in choosing the right drug and adjust its dose in specific patients. To this end, we aimed to assess time patterns of risperidone and its metabolite, 9-hydroxyrisperidone, in children and adolescents with oppositional defiant and/or conduct disorder.

Methods: We measured plasma concentrations of risperidone and 9-hydroxyrisperidone, their sum (active moiety, AM) and ratio, as well as plasma concentrations corrected for daily dose (C/D), from 140 children/adolescents with the above-mentioned disorders. We used Student's $t$ test to compare females versus males, patients under versus over 16-year-old, patients with lower versus higher than the median body weight, and patients with lower versus higher than the median body mass index (BMI). Two mixed-effects logistic regression models were fitted for risperidone/9hydroxyrisperidone ratio and AM, respectively, by considering risperidone daily dose and patients' demographic characteristics.

Results: Females had higher 9-hydroxyrisperidone and AM plasma concentrations than males $(p=0.004$ and $p=0.034)$. Younger patients had lower risperidone plasma concentration and risperidone/9-hydroxyrisperidone ratio $(p=0.02$ and $p=0.021)$, but higher C/D 9-hydroxyrisperidone and AM than older patients $(p=0.013$ and $p=0.043)$. Lower-weight patients had lower plasma risperidone and risperidone/9-hydroxyrisperidone ratio $(p=0.014$ and $p=0.019)$, but higher C/D 9-hydroxyrisperidone concentration than heavier patients $(p=0.03)$. All these results could be accounted for by daily dose. Patients with lower and higher BMI did not differ significantly. Regression analyses showed that only risperidone daily dose predicted risperidone/9-hydroxyrisperidone ratio, whereas risperidone daily dose, sex, and age predicted AM.

Conclusion: Clinicians prescribing risperidone need to consider sex, age, and weight, but not BMI when adjusting daily doses.

KEY WORDS: Oppositional defiant disorder; Conduct disorder; Youth; Risperidone; 9-Hydroxyrisperidone; Therapeutic drug monitoring.
\end{abstract}

Received: April 22, 2019 / Revised: September 1, 2019

Accepted: September 6, 2019

Address for correspondence: Daria Piacentino

Section on Clinical Psychoneuroendocrinology and

Neuropsychopharmacology, National Institute on Alcohol Abuse

and Alcoholism (NIAAA), Division of Intramural Clinical and

Basic Research and National Institute on Drug Abuse (NIDA),

Intramural Research Program, National Institutes of Health (NIH),

10 Center Drive, Bethesda, MD 20892, USA

E-mail: daria.piacentino@nih.gov

ORCID: https://orcid.org/0000-0002-9991-7403

\section{INTRODUCTION}

Oppositional defiant disorder (ODD) and conduct disorder (CD) are common disruptive, impulse-control, and CDs in children and adolescents that could constitute a continuum, as ODD may precede CD and be a risk factor for its development [1]. They are externalizing disorders sharing many features with attention deficit/hyperactivity

(c) This is an Open-Access article distributed under the terms of the Creative Commons Attribution Non-Commercial License (http://creativecommons.org/licenses/by-nc/4.0) which permits unrestricted non-commercial use, distribution, and reproduction in any medium, provided the original work is properly cited. 
disorder (ADHD), but also having distinctive neuroanatomical characteristics [2,3]. In the US, lifetime prevalence is about $10 \%$ for ODD and slightly less for CD, with boys affected slightly more by ODD and considerably so by $\mathrm{CD}[4,5]$.

An essential part of the psychiatric care for pediatric disruptive/impulsive/CDs includes pharmacotherapy, despite the lack of Food and Drug Administration (FDA)-approved medications. In fact, there is considerable evidence from off-label prescriptions suggesting a role for several antipsychotic drugs, including risperidone [6-10]. Risperidone obtained FDA-approval for the treatment of irritability in children and adolescents with autism spectrum disorders. In several European countries, it is also approved for children and adolescents with $C D$, in the presence of intellectual hypofunctioning and when all other strategies have failed [11].

The primary pathway of risperidone (risperidone plasma concentration, RIS) metabolism is a CYP2D6-mediated 9-hydroxylation leading to its main active metabolite, 9-hydroxyrisperidone (9-OH-RIS) [12]. Additional pathways may include CYP3A4 and CYP3A5 [13,14]. As 9-OH-RIS has approximately $70 \%$ of the pharmacological activity of RIS [15], clinicians consider the sum of concentration for RIS and 9-OH-RIS (active moiety, AM). The Arbeitsgemeinschaft für Neuropsychopharmakologie und Pharmakopsychiatrie (AGNP) consensus guidelines on therapeutic drug monitoring (TDM) suggest a therapeutic reference range of $20-60 \mathrm{ng} / \mathrm{ml}$ for $\mathrm{AM}$ in adult patients [12].

TDM is a specific method of clinical pharmacology used to guide clinical routine psychopharmacotherapy based on the measurement of blood drug concentrations. The amount of pharmacokinetic data from RIS-medicated children and adolescents is disproportionately smaller compared to adults. In fact, data mainly derive from children and adolescents with autism spectrum disorders [16-21], while patients with other indications for RIS treatment are underrepresented [22,23]. Furthermore, the available data demonstrate a smaller interindividual variability of RIS concentrations in children and adolescents compared to adults [24]. Thus, we expected a narrower therapeutic reference range for children and adolescents. The clinical utility of measuring RIS concentration in these patients is reflected on the correlation between prolactin elevation and concentration of 9-OH-RIS [16,17].
To our knowledge, there is dearth of data on RIS pharmacokinetics in patients with disruptive/impulsive/CDs. Thus, the aim of this study was to assess the plasma concentration curves of RIS, its active metabolite, and their total (AM) in RIS-treated children and adolescents affected by the above-mentioned disorders, with a focus on the role of drug dose and patients' demographics.

\section{METHODS}

\section{Patients}

In this observational retrospective cohort study, 140 children and adolescents with disruptive/impulsive/CDs (89 males, age range $=5-18$ years; 63 females, age range $=$ 7-18 years) were consecutively recruited during 2014 to 2016 as part of the clinical routine at the Department of Psychiatry of the Central Hospital of Bolzano/Bozen (Italy), at the Department of Psychiatry of the University Clinic of Innsbruck (Austria), and at the Department of Psychiatry and Psychotherapy of the University of Regensburg (Germany). Diagnosis of ODD and/or CD was posed according to the Diagnostic and Statistical Manual of Mental Disorders, 5th edition (DSM-5) [25] criteria by treating clinicians, who made drug prescription. The aim of the study was thoroughly explained before obtaining parental written informed consent and assent of children or adolescents. This was a prerequisite to perform the study and publish results. The study fully respected the Ethical Principles for Medical Research Involving Human Subjects, as adopted by the 18th World Medical Association General Assembly (WMA GA), Helsinki, Finland, June 1964, and subsequently amended by the 64th WMA GA, Fortaleza, Brazil, October 2013. Patient anonymity was guaranteed.

Body weight and height were measured based on standard procedures; body mass index (BMI) was calculated accordingly. We excluded patients receiving concomitant medication with potential CYP2D6 or CYP3A4 inhibitors or inducers [12]. Only five patients received other psychotropic drugs during the study period, three lorazepam and two oxazepam. Samples with missing data of RIS or its metabolite were not included in the analysis. In the case of multiple available plasma concentrations for a single patient, only the most recent steady-state plasma concentration was included in the analyses. 


\section{Quantification of Risperidone and 9-Hydroxyrisperidone}

Clinicians were instructed to draw blood under trough conditions (before drug administration) at steady state ( $>5$ elimination half-lives under the same drug dose). In all three hospitals, the same analytical method was used; RIS and 9-OH-RIS concentrations were determined by using high performance liquid chromatography with ultraviolet detection (HPLC/UV) [26]. The method was validated according to DIN 32645 (Deutsche Industrie Norm 32645, described in guidelines of GTFCh (Society of Toxicology and Forensic Chemistry) in consideration of ISO 5725 (International Organization for Standardization) [27], US FDA guidance [28], and ICH (International Conference on Harmonization) requirements [29]. The laboratories involved in the study regularly run internal quality controls and participated in INSTAND-external quality assessment schemes (Düsseldorf, Germany; uww.instandev.de). The limit of detection, defined as signal-to-noise ratio of 3:1, was $0.5 \mathrm{ng} / \mathrm{ml}$ for RIS and 9-OH-RIS. The lower limit of quantification (LLOQ) was $1 \mathrm{ng} / \mathrm{ml}$ for RIS and 9-OH-RIS. The interday precision, determined as duplicates on three different days at $1 \mathrm{ng} / \mathrm{ml}$, was $5.56 \%$ of mean and $5.21 \%$ of mean, respectively. However, the analytical process that uses HPLC/UV also detects both RIS and 9-OH-RIS values below the LLOQ. In two patients, RIS concentration was $0.6 \mathrm{ng} / \mathrm{ml}$ in one and $0.8 \mathrm{ng} / \mathrm{ml}$ in the other, while 9-OH-RIS concentrations were 15 and 21 $\mathrm{ng} / \mathrm{ml}$, respectively. The potential bias of including these RIS values (instead of replacing with the LLOQ) in our models is expected to be minimal, thus we decided to include these defined values in our analysis, as they were true values above zero.

\section{Statistical Analysis}

Data distribution was normal (Shapiro-Wilk test, $W=$ 0.835, $p>0.05)$, thus we proceeded with parametric testing. Continuous variables (e.g., age, BMI, risperidone daily dose, RIS, 9-OH-RIS) were expressed as mean ( $\bar{x})$ and standard deviations. Categorical variables (i.e., sex) were expressed as absolute and percentage values. Comparisons between sample groups, i.e., males vs. females, younger vs. older patients (median, age $\leq 15$ years vs. age of $16-18$ years), patients with lighter vs. heavier weight (median, $\leq 55 \mathrm{~kg}$ vs. $>55 \mathrm{~kg}$ ), and patients with lower vs. higher BMI (median, $\leq 20.7 \mathrm{~kg} / \mathrm{m}^{2}$ vs. $>20.7$ $\mathrm{kg} / \mathrm{m}^{2}$ ), were assessed with the parametric Student's in- dependent $t$ test. Correlations were performed with Spearman's $r$. The cut-off for statistical significance was set at $p<0.05$ (two-tailed). We fitted two Bonferroni-adjusted mixed effects logistic regression models where our dependent variables were RIS/9-OH-RIS and AM, respectively, and our independent variables were RIS daily dose and patients' demographic characteristics, both continuous and categorical (i.e., sex, age, weight, BMI). Bonferroni adjustment was used in our multiple hypothesis testing to maintain control of the false discovery rate while incorporating prior information about the hypotheses. The prior information takes the form of $p$ value weights. If the assignment of weights is positively associated with the null hypotheses being false, the procedure improves power, except in cases where power is already near one. Even if the assignment of weights is poor, power is only reduced slightly, as long as the weights are not too large [30]. Nagelkerke's pseudo- $R^{2}$ was calculated to determine the proportion of the variance in the dependent variables (RIS/9-OH-RIS ratio and AM) which is predictable from the independent variables. Nagelkerke's pseudo- $R^{2}$ is the percentage of the response variable variation that is explained by a linear model $\left(R^{2}=\right.$ explained variation/total variation). $R^{2}$ is always between 0 and 1 , with 0 indicating that the model explains none of the variability of the response data around its mean and 1 indicating that the model explains all the variability of the response data around its mean. Analyses were performed by using the Statistical Package for the Social Sciences software (IBM SPSS Statistics for Windows ver. 24.0, 2016; IBM Corp., Armonk, NY, USA).

\section{RESULTS}

The sample of 140 patients consisted of 81 boys and 59 girls. Of them, 64 (45.7\%) were diagnosed with ODD only, 53 (37.9\%) with CD only, and 23 (16.4\%) with comorbid ODD/CD. By method we excluded patients receiving concomitant medication with potential CYP2D6 or CYP3A4 inhibitors or inducers; the five patients taking concomitant medication presented no such problem. The demographic and pharmacokinetic characteristics are shown in Table 1.

First, we compared males vs. females for plasma and corrected for daily does (C/D) plasma concentrations for RIS, 9-OH-RIS, AM as well as RIS/9-OH-RIS ratios, by us- 
Table 1. Patients' demographic and pharmacokinetic characteristics $(n=140)$

\begin{tabular}{lc}
\hline \multicolumn{1}{c}{ Variable } & Value \\
\hline Sex & \\
$\quad$ Male & $51(57.86)$ \\
$\quad$ Female & $59(42.14)$ \\
Age (yr) & $14.2 \pm 3.1(5.0-18.0)$ \\
Weight $(\mathrm{kg})$ & $53.92 \pm 16.23(20.10-124.02)$ \\
Height $(\mathrm{m})$ & $1.59 \pm 0.15(1.09-1.92)$ \\
BMI $\left(\mathrm{kg} / \mathrm{m}^{2}\right)$ & $20.66 \pm 3.12(15.03-33.63)$ \\
Risperidone daily dose (mg/day) & $1.5 \pm 1.0(0.5-7.0)$ \\
RIS (ng/ml) & $6.41 \pm 9.91(0.1-58.0)$ \\
9-OH-RIS (ng/ml) & $15.96 \pm 14.63(1.0-113.0)$ \\
AM & $22.37 \pm 18.72(2.0-114.5)$ \\
RIS/9-OH-RIS ratio & $0.51 \pm 1.04(0.003-5.31)$ \\
C/D RIS (ng/ml) (mg/day) & $4.46 \pm 5.61(0.07-29.64)$ \\
C/D 9-OH-RIS (ng/ml) (mg/day) & $16.33 \pm 25.51(0.33-196.02)$ \\
C/D AM (ng/ml) (mg/day) & $20.91 \pm 26.62(0.67-228.12)$ \\
\hline
\end{tabular}

Values are presented as number (\%) or mean \pm standard deviation (range).

BMI, body mass index; RIS, risperidone plasma concentration; 9OH-RIS, 9-hydroxyrisperidone plasma concentration; AM, active moiety; C/D, dose-corrected plasma concentration. ing Student's $t$ test. Similarly, by using the median as the cut-off value, we compared younger ( $\leq 15$ years) vs. older $(16-18$ years) patients, patients with lighter $(\leq 55 \mathrm{~kg})$ vs. heavier ( $>55 \mathrm{~kg}$ ) weight, and patients with lower $(\leq 20.7$ $\mathrm{kg} / \mathrm{m}^{2}$ ) vs. higher (> $20.7 \mathrm{~kg} / \mathrm{m}^{2}$ ) BMI (Table 2).

Females had higher 9-OH-RIS and AM plasma concentrations than males $(t=2.91, p=0.004$ and $t=2.15, p=$ 0.034 , respectively). Younger patients had lower RIS daily doses, RIS plasma concentrations, and RIS/9-OH-RIS ratios $(t=4.651, p=0.001, t=-2.36, p=0.02$, and $t=-$ 2.43, $p=0.021$, respectively), but higher C/D 9-OH-RIS and C/D AM compared to older patients ( $t=2.54, p=$ 0.013 and $t=2.05, p=0.043$, respectively). Patients with lighter weight had lower RIS daily doses, RIS plasma concentrations, and RIS/9-OH-RIS ratios $(t=-3.67, p=$ $0.001, t=-2.51, p=0.014$, and $t=-2.24, p=0.019$, respectively), but higher $\mathrm{C} / \mathrm{D} 9-\mathrm{OH}-\mathrm{RIS}$ concentrations than patients with heavier weight $(t=2.20, p=0.03)$. No significant differences were found between patients with lower and higher BMI, with the exception of RIS daily doses $(t=-3.321, p=0.001)$.

Table 2. Comparison of plasma and C/D plasma concentrations for RIS, 9-OH-RIS, AM as well as RIS/9-OH-RIS ratios between males vs. females, younger vs. older patients, patients with lower vs. higher weight, and patients with lower vs. higher BMI

\begin{tabular}{|c|c|c|c|c|c|c|c|c|}
\hline Variable & $\begin{array}{l}\text { RIS daily } \\
\text { dose }\end{array}$ & $\begin{array}{c}\text { RIS plasma } \\
\text { concentration }\end{array}$ & $\begin{array}{c}\text { 9-OH-RIS } \\
\text { plasma } \\
\text { concentration }\end{array}$ & $\begin{array}{c}\text { RIS/9-OH-RI } \\
\text { S ratio }\end{array}$ & AM & $\begin{array}{l}\text { C/D RIS } \\
\text { (ng/ml) } \\
\text { (mg/day) }\end{array}$ & $\begin{array}{c}\text { C/D 9-OH-RIS } \\
\text { (ng/ml) } \\
\text { (mg/day) }\end{array}$ & $\begin{array}{l}\text { C/D AM } \\
(\mathrm{ng} / \mathrm{ml}) \\
\text { (mg/day) }\end{array}$ \\
\hline \multicolumn{9}{|l|}{ Males vs. females } \\
\hline Male $(n=81)$ & $1.34 \pm 0.80$ & $6.07 \pm 9.91$ & $13.32 \pm 14.16$ & $0.46 \pm 0.10$ & $19.12 \pm 17.98$ & $4.53 \pm 5.87$ & $13.57 \pm 25.32$ & $18.01 \pm 25.96$ \\
\hline Female $(n=59)$ & $1.46 \pm 0.92$ & $6.94 \pm 10.01$ & $20.17 \pm 14.42$ & $0.35 \pm 1.22$ & $25.62 \pm 17.41$ & $4.62 \pm 5.30$ & $20.21 \pm 25.54$ & $24.92 \pm 27.37$ \\
\hline$t$ & 0.813 & 0.48 & 2.91 & 0.46 & 2.15 & 0.09 & 1.55 & 1.5 \\
\hline$p$ value & 0.417 & 0.634 & $0.004 *$ & 0.528 & $0.034^{*}$ & 0.927 & 0.123 & 0.136 \\
\hline \multicolumn{9}{|l|}{ Younger vs. older age } \\
\hline$\leq 15 \mathrm{yr}(\mathrm{n}=79)$ & $1.11 \pm 0.69$ & $4.62 \pm 7.44$ & $16.01 \pm 17.17$ & $0.25 \pm 0.37$ & $19.35 \pm 2.21$ & $3.95 \pm 4.70$ & $20.61 \pm 32.52$ & $24.59 \pm 33.57$ \\
\hline $16-18 \mathrm{yr}(\mathrm{n}=61)$ & $1.75 \pm 0.91$ & $8.86 \pm 12.12$ & $15.91 \pm 10.75$ & $0.56 \pm 0.78$ & $24.73 \pm 17.85$ & $5.37 \pm 6.66$ & $10.83 \pm 9.03$ & $16.25 \pm 12.14$ \\
\hline$t$ & 4.651 & -2.36 & 0.04 & -2.43 & -1.29 & -1.42 & 2.54 & 2.05 \\
\hline$p$ value & $0.001 *$ & $0.02^{*}$ & 0.968 & $0.021^{*}$ & 0.2 & 0.158 & $0.013^{*}$ & $0.043^{*}$ \\
\hline \multicolumn{9}{|l|}{ Lighter vs. heavier weight } \\
\hline$\leq 55 \mathrm{~kg}(\mathrm{n}=80)$ & $1.17 \pm 0.71$ & $4.51 \pm 7.36$ & $16.43 \pm 16.71$ & $0.24 \pm 0.59$ & $21.14 \pm 18.32$ & $3.78 \pm 4.38$ & $20.01 \pm 31.61$ & $23.78 \pm 32.89$ \\
\hline$>55 \mathrm{~kg}(\mathrm{n}=60)$ & $1.68 \pm 0.94$ & $9.14 \pm 12.11$ & $15.31 \pm 11.23$ & $0.60 \pm 1.01$ & $24.17 \pm 18.34$ & $5.61 \pm 6.83$ & $11.52 \pm 12.32$ & $17.11 \pm 14.35$ \\
\hline$t$ & -3.67 & -2.51 & 0.52 & -2.24 & -0.43 & -1.81 & 2.2 & 1.63 \\
\hline$p$ value & $0.001^{*}$ & $0.014^{*}$ & 0.606 & $0.019 *$ & 0.671 & 0.073 & $0.03 *$ & 0.105 \\
\hline \multicolumn{9}{|l|}{ Lower vs. higher BMI } \\
\hline$\leq 20.7 \mathrm{~kg} / \mathrm{m}^{2}(\mathrm{n}=75)$ & $1.17 \pm 0.68$ & $4.91 \pm 7.63$ & $16.83 \pm 17.21$ & $0.29 \pm 1.13$ & $21.74 \pm 19.62$ & $4.07 \pm 4.48$ & $20.01 \pm 32.51$ & $24.09 \pm 33.69$ \\
\hline$>20.7 \mathrm{~kg} / \mathrm{m}^{2}(\mathrm{n}=65)$ & $1.64 \pm 0.96$ & $8.14 \pm 11.81$ & $14.97 \pm 10.93$ & $0.54 \pm 0.22$ & $23.17 \pm 17.84$ & $5.14 \pm 6.68$ & $12.12 \pm 12.62$ & $17.26 \pm 14.31$ \\
\hline$t$ & -3.321 & -1.89 & 0.79 & -1.99 & -0.43 & -1.1 & 1.95 & 1.6 \\
\hline$p$ value & $0.001^{*}$ & 0.058 & 0.432 & 0.068 & 0.671 & 0.274 & 0.058 & 0.112 \\
\hline
\end{tabular}

Values are presented as mean \pm standard deviation.

C/D, dose-corrected plasma concentration; RIS, risperidone plasma concentration; 9-OH-RIS, 9-hydroxyrisperidone plasma concentration; AM, active moiety; BMI, body mass index.

An asterisk indicates the level of significance $(p<0.05)$. 
Table 3. Mixed effects logistic regression with RIS/9-OH-RIS ratio as a dependent variable

\begin{tabular}{lcccc}
\hline \multicolumn{1}{c}{ Source } & $\begin{array}{c}\text { Numerator } \\
\mathrm{df}\end{array}$ & $\begin{array}{c}\text { Denominator } \\
\mathrm{df}\end{array}$ & $\mathrm{F}$ & $p$ value \\
\hline Intercept & 1 & 16 & 2.497 & 0.134 \\
Sex & 1 & 16 & 2.964 & 0.104 \\
Age & 6 & 16 & 1.361 & 0.289 \\
Weight & 1 & 16 & 0.670 & 0.425 \\
BMl & 54 & 16 & 0.860 & 0.673 \\
RIS daily dose & 4 & 16 & 3.409 & $0.017^{\mathrm{a} *}$ \\
\hline
\end{tabular}

BMI, body mass index; RIS, risperidone plasma concentration; 9$\mathrm{OH}-\mathrm{RIS}$, 9-hydroxyrisperidone plasma concentration; df, degree of freedom.

${ }^{\mathrm{a}}$ After Bonferroni correction. An asterisk indicates the level of significance $(p<0.05)$.

The Pearson correlation coefficient showed the strength of the association between RIS daily dose and RIS $(r=0.298, p=0.04)$ and 9-OH-RIS $(r=0.355, p=0.01)$ plasma concentrations, respectively.

In the first Bonferroni-corrected mixed effects logistic regression model (Table 3), we found only one variable, i.e., RIS daily dose, to be predictive of RIS/9-OH-RIS ratio. We have also performed the same model after removing the two patients who had a RIS concentration of 0.6 and $0.8 \mathrm{ng} / \mathrm{ml}$, respectively, and we have found no differences in significances, as expected. Nagelkerke's pseudo- $R^{2}$ was 0.181 , meaning that our regression model explains $18.1 \%$ of the variance of the dependent variable.

In the second Bonferroni-corrected mixed effects logistic regression model (Table 4), we found three variables, i.e., RIS daily dose and patients' sex and age to be predictive of AM. Weight approached but did not quite achieve significance $(p=0.056)$. Nagelkerke's pseudo- $R^{2}$ was 0.364 , meaning that our regression model explains $36.4 \%$ of the variance of the dependent variable.

\section{DISCUSSION}

In this study, we sought to investigate the association between daily risperidone doses, as well as patients' demographic characteristics, and pharmacokinetic parameters of risperidone. Sex has a substantial effect on risperidone metabolism. Sex differences for risperidone metabolism in adolescents have been previously poorly investigated, as data mainly derived from patients with autism spectrum disorders, where males were overrepresented [16-18,23]. Nevertheless, our findings are aligned with
Table 4. Mixed effects logistic regression with $\mathrm{AM}$ as a dependent variable

\begin{tabular}{lcccl}
\hline \multicolumn{1}{c}{ Source } & $\begin{array}{c}\text { Numerator } \\
\text { df }\end{array}$ & $\begin{array}{c}\text { Denominator } \\
\text { df }\end{array}$ & $F$ & $p$ value \\
\hline Intercept & 1 & 16 & 20.008 & 0.000 \\
Sex & 1 & 16 & 1.923 & $0.015^{*}$ \\
Age & 6 & 16 & 1.867 & $0.049^{*}$ \\
Weight & 1 & 16 & 0.798 & 0.056 \\
BMI & 54 & 16 & 1.442 & 0.260 \\
RIS daily dose & 4 & 16 & 2.734 & $0.001^{\mathrm{a} *}$ \\
\hline
\end{tabular}

$\mathrm{AM}$, active moiety; $\mathrm{BMI}$, body mass index; RIS, risperidone plasma concentration; $\mathrm{df}$, degree of freedom.

${ }^{a}$ After Bonferroni correction. An asterisk indicates the level of significance $(p<0.05)$.

early data reporting enhanced levels in females, compared with males, in a study that adjusted for dose [22]. Differences for risperidone concentrations between males and females with other psychiatric diagnoses have been previously explained in light of sex-dependent CYP3A4 activity patterns [31]. This explanation appears plausible in our sample, as differences for AM were driven by differences for the higher active metabolite concentrations in females compared to males. Therefore, we may hypothesize that prominent sex-specific differences for CYP3A4 activity are already present in adolescents, as RIS daily dose did not differ between the two sexes (Table 2). Still, the sex-specific patterns reported in our sample may at least partially reflect dose differences, but only secondarily, as boys tend to be heavier than girls and did not persist after controlling for drug dose.

Of particular interest are the findings regarding the effects of body composition on risperidone metabolism. Our analyses supported significant effects for weight, but not BMI. In fact, we found higher RIS/9-OH-RIS ratios in older vs. younger patients and in heavier vs. lighter body weight patients, while the effect of higher vs. lower BMI was much weaker at this respect and present only as a trend (Table 2), meaning that as patients grow and their weight increases, their rate of conversion of RIS to 9-OH-RIS slows down. In the above groupings, it is interesting to underline that dosing differed significantly, while it did not in the male vs. female comparison. This notion contrasts previous findings of RIS adult pharmacokinetics, where BMI and not weight displayed crucial effects [32]. Thus, our knowledge of adults RIS pharmacokinetics cannot be automatically transferred to children and adolescents. It seems that, unlike adults, BMI does not 
precisely reflect pharmacokinetically-relevant parameters such as drug distribution volume in children [32]. On the other hand, weight seems to be crucial; low-weight patients had lower ratios and higher $\mathrm{C} / \mathrm{D}$ 9-OH-RIS concentrations than patients with higher weight. Such differences could be accounted for by dosing, as clinicians tend to prescribe higher doses to older children and older children tend to weigh more than younger children. Lipophilic agents such as risperidone and 9-OH-RIS may be detected in higher concentrations in blood of patients with lower fat percentages.

Since AM reflects the total circulating level of RIS and 9-OH-RIS, while the RIS/9-OH-RIS ratio is the fraction of RIS to its metabolite, which is also marketed as the antipsychotic paliperidone, and has been found to be safe and effective in first-episode schizophrenia [33], and given that the latter ratio was predicted by our mixed effects logistic regression models only by RIS daily dose, while AM was predicted by most measures entered in the mod$\mathrm{el}$, including RIS daily dose, which was the most significantly associated parameter in the model, RIS daily dose is the measure that should most be considered in treating patients with disruptive/impulsive/CDs of pediatric age.

Our evidence points to distinct pharmacokinetic patterns for risperidone and 9-OH-RIS plasma concentrations in children and adolescents with disruptive/impulsive/CDs. RIS prescription in these patients remains off-label and clinicians may use TDM to adjust doses and prevent accumulation phenomena. Sex, age, and weight in priority to BMI should be considered by clinicians in their search to find the right RIS dose.

\section{Limitations}

Our data derive from a naturalistic sample assessed retrospectively; this might create reliability issues. Moreover, we had no knowledge about onset and duration of illness, psychometric and safety data, and comorbidities; this prevented us from conducting further analyses. The age range we have considered is one of transition of $\mathrm{P} 450 \mathrm{cy}$ tochrome function, which cannot be adequately assessed without genotyping or phenotyping. The inclusion of genetic data could have provided additional insight [20]. We here did not attempt to correlate our TDM data with clinical response or daily dose, but doses do not correlate with plasma drug or metabolite concentrations with this drug [34]. However, risperidone is effectively used in pediatric age populations $[35,36]$, as are other antipsychotics in the conditions considered here [37].

\section{Conclusions}

In this study we provided data on risperidone metabolism in children and adolescents with disruptive/impulsive/CDs. We found RIS plasma concentrations and ratios to be lower in younger vs. older and lighter vs. heavier pediatric patients, while BMI had no significant effect, but these differences might be due to different (and lower) dosing; sex was significantly associated with 9-OH-RIS plasma concentrations, but not AM. The stronger correlation between daily risperidone dose and 9-OH-RIS plasma concentrations, compared with risperidone plasma concentration, suggests a rapid conversion of the drug into its metabolite, with its significantly longer half-life. Hence dosing should be approached considering sex-, age- and weight-related risperidone disposition patterns.

\section{- Conflicts of Interest}

No potential conflict of interest relevant to this article was reported.

\section{Author Contributions}

DP, GDK, GS, MG, and AC contributed equally to this work. DP and AC designed research. DP, GS, MP, and $M G$ performed acquisition of data. DP planned and performed the statistical analysis. DP, GS, EH, SC, MG, and AC wrote the manuscript; GDK, GG, SC, MG, and AC revised the manuscript. All authors read and approved the final manuscript.

\section{- ORCID}

Daria Piacentino https://orcid.org/0000-0002-9991-7403 Georgios D. Kotzalidis

https://orcid.org/0000-0002-0281-6324

Georgios Schoretsanitis

https://orcid.org/0000-0002-3851-4117

Michael Paulzen https://orcid.org/0000-0003-4198-5160

Ekkehard Haen https://orcid.org/0000-0003-4430-4148

Simone Cappelletti https://orcid.org/0000-0002-9331-4273

Giancarlo Giupponi https://orcid.org/0000-0001-6430-0000

Michael Grözinger https://orcid.org/0000-0001-8700-9646

Andreas Conca https://orcid.org/0000-0003-4841-7510 


\section{REFERENCES}

1. Rowe R, Maughan B, Pickles A, Costello EJ, Angold A. The relationship between DSM-IV oppositional defiant disorder and conduct disorder: findings from the Great Smoky Mountains study. J Child Psychol Psychiatry 2002;43:365-373.

2. Noordermeer SD, Luman M, Oosterlaan J. A systematic review and meta-analysis of neuroimaging in oppositional defiant disorder $(O D D)$ and conduct disorder $(C D)$ taking attention-deficit hyperactivity disorder (ADHD) into account. Neuropsychol Rev 2016;26:44-72.

3. Sasayama D, Hayashida A, Yamasue H, Harada Y, Kaneko T, Kasai K, et al. Neuroanatomical correlates of attention-deficit-hyperactivity disorder accounting for comorbid oppositional defiant disorder and conduct disorder. Psychiatry Clin Neurosci 2010;64:394-402.

4. Nock MK, Kazdin AE, Hiripi E, Kessler RC. Prevalence, subtypes, and correlates of DSM-IV conduct disorder in the National Comorbidity Survey Replication. Psychol Med 2006;36:699-710.

5. Nock MK, Kazdin AE, Hiripi E, Kessler RC. Lifetime prevalence, correlates, and persistence of oppositional defiant disorder: results from the National Comorbidity Survey Replication. J Child Psychol Psychiatry 2007;48:703-713.

6. Gadow KD, Arnold LE, Molina BS, Findling RL, Bukstein OG, Brown NV, et al. Risperidone added to parent training and stimulant medication: effects on attention-deficit/hyperactivity disorder, oppositional defiant disorder, conduct disorder, and peer aggression. J Am Acad Child Adolesc Psychiatry 2014; 53:948-959.e1.

7. Harrison JN, Cluxton-Keller F, Gross D. Antipsychotic medication prescribing trends in children and adolescents. J Pediatr Health Care 2012;26:139-145.

8. Persico AM, Arango C, Buitelaar JK, Correll CU, Glennon JC, Hoekstra PJ, et al. Unmet needs in paediatric psychopharmacology: Present scenario and future perspectives. Eur Neuropsychopharmacol 2015;25:1513-1531.

9. Pringsheim T, Hirsch L, Gardner D, Gorman DA. The pharmacological management of oppositional behaviour, conduct problems, and aggression in children and adolescents with attention-deficit hyperactivity disorder, oppositional defiant disorder, and conduct disorder: a systematic review and meta-analysis. Part 1: psychostimulants, alpha-2 agonists, and atomoxetine. Can J Psychiatry 2015;60:42-51.

10. Simeon J, Milin R, Walker S. A retrospective chart review of risperidone use in treatment-resistant children and adolescents with psychiatric disorders. Prog Neuropsychopharmacol Biol Psychiatry 2002;26:267-275.

11. Nesvåg R, Hendset M, Refsum H, Tanum L. Serum concentrations of risperidone and 9-OH risperidone following intramuscular injection of long-acting risperidone compared with oral risperidone medication. Acta Psychiatr Scand 2006;114: 21-26.

12. Hiemke C, Bergemann N, Clement HW, Conca A, Deckert J,
Domschke K, et al. Consensus guidelines for therapeutic drug monitoring in neuropsychopharmacology: update 2017. Pharmacopsychiatry 2018;51:9-62.

13. Xiang Q, Zhao X, Zhou Y, Duan JL, Cui YM. Effect of CYP2D6, CYP3A5, and MDR1 genetic polymorphisms on the pharmacokinetics of risperidone and its active moiety. J Clin Pharmacol 2010;50:659-666.

14. Yasui-Furukori N, Hidestrand M, Spina E, Facciolá G, Scordo MG, Tybring G. Different enantioselective 9-hydroxylation of risperidone by the two human CYP2D6 and CYP3A4 enzymes. Drug Metab Dispos 2001;29:1263-1268.

15. Heykants J, Huang ML, Mannens G, Meuldermans W, Snoeck E, Van Beijsterveldt L, et al. The pharmacokinetics of risperidone in humans: a summary. J Clin Psychiatry 1994;55 Suppl:13-17.

16. Ngamsamut $N$, Hongkaew $Y$, Vanwong $N$, Srisawasdi $P$, Puangpetch A, Chamkrachangpada B, et al. 9-Hydroxyrisperidone-induced hyperprolactinaemia in Thai children and adolescents with Autism Spectrum Disorder. Basic Clin Pharmacol Toxicol 2016;119:267-272.

17. Nuntamool N, Ngamsamut N, Vanwong N, Puangpetch A, Chamnanphon $\mathrm{M}$, Hongkaew $\mathrm{Y}$, et al. Pharmacogenomics and efficacy of risperidone long-term treatment in Thai autistic children and adolescents. Basic Clin Pharmacol Toxicol 2017;121:316-324.

18. Roke Y, Buitelaar JK, Boot AM, Tenback D, van Harten PN. Risk of hyperprolactinemia and sexual side effects in males 10-20 years old diagnosed with autism spectrum disorders or disruptive behavior disorder and treated with risperidone. I Child Adolesc Psychopharmacol 2012;22:432-439.

19. Roke Y, van Harten PN, Franke B, Galesloot TE, Boot AM, Buitelaar JK. The effect of the Taq1A variant in the dopamine $D_{2}$ receptor gene and common CYP2D6 alleles on prolactin levels in risperidone-treated boys. Pharmacogenet Genomics 2013;23:487-493.

20. Sukasem C, Hongkaew Y, Ngamsamut N, Puangpetch A, Vanwong N, Chamnanphon M, et al. Impact of pharmacogenetic markers of CYP2D6 and DRD2 on prolactin response in risperidone-treated Thai children and adolescents with autism spectrum disorders. J Clin Psychopharmacol 2016;36: 141-146.

21. Vanwong N, Ngamsamut N, Medhasi S, Puangpetch A, Chamnanphon M, Tan-Kam T, et al. Impact of CYP2D6 polymorphism on steady-state plasma levels of risperidone and 9-hydroxyrisperidone in Thai children and adolescents with autism spectrum disorder. J Child Adolesc Psychopharmacol 2017;27:185-191.

22. Aichhorn W, Marksteiner J, Walch T, Zernig G, Hinterhuber $\mathrm{H}$, Stuppaeck C, et al. Age and gender effects on olanzapine and risperidone plasma concentrations in children and adolescents. JChild Adolesc Psychopharmacol 2007; 17:665674.

23. Calarge CA, Miller del D. Predictors of risperidone and 9-hy- 
droxyrisperidone serum concentration in children and adolescents. JChild Adolesc Psychopharmacol 2011;21:163169.

24. Balant-Gorgia AE, Gex-Fabry M, Genet C, Balant LP. Therapeutic drug monitoring of risperidone using a new, rapid HPLC method: reappraisal of interindividual variability factors. Ther Drug Monit 1999;21:105-115.

25. American Psychiatric Association. Diagnostic and statistical manual of mental disorders. 5th ed. Arlington:American Psychiatric Publishing;2013.

26. Bader W, Melchner D, Nonenmacher T, Haen E. Determination of five commonly used antipsychotics in human serum by high performance-liquid chromatography (HPLC) and electrochemical detection. Pharmacopsychiatry 2005;38.

27. Paul LD, Mußhof F, Aebi B, Auwärter V, Krämer T, Peters FT, et al. [Richtlinie der GTFCh zur qualitätssicherung bei forensisch-toxikologischen untersuchungen]. Toxichem Krimtech 2009;76:142-176. German.

28. U.S. Food \& Drug Administration. Bioanalytical method validation guidance for industry [Internet]. Silver Spring: U.S. Food \& Drug Administration; 2001 lupdated 2018 May 18; cited at 2019 Aug 3]. Available from: http://www.fda.gov/ ucm/groups/fdagov-public/@fdagov-drugs-gen/documents/document/ucm070107.pdf.

29. ICH International Conference on harmonization. ICH Harmonised tripartite guideline. Validation of analytical procedures: test and methodology Q2(R1). In: Abstracts of the 3rd International Conference on Harmonisation of Technical Requirements for Registration of Pharmaceuticals for Human Use; Jul 18, 1996; Paris, France. Abstract NR 244:519.

30. Genovese CR, Roeder K, Wasserman L. False discovery control with p-value weighting. Biometrika 2006;93:509-524.
31. Schoretsanitis G, Spina E, Hiemke C, de Leon J. A systematic review and combined analysis of therapeutic drug monitoring studies for long-acting risperidone. Expert Rev Clin Pharmacol 2017;10:965-981.

32. Paulzen M, Haen E, Stegmann B, Hiemke C, Gründer G, Lammertz SE, et al. Body mass index (BMI) but not body weight is associated with changes in the metabolism of risperidone; A pharmacokinetics-based hypothesis. Psychoneuroendocrinology 2016;73:9-15.

33. Kang NI, Koo BH, Kim SW, Kim JH, Nam B, Lee BJ, et al. Efficacy and tolerability of paliperidone extended-release in the treatment of first-episode psychosis: an eight-week, openlabel, multicenter trial. Clin Psychopharmacol Neurosci 2016;14:261-269.

34. Lostia AM, Mazzarini L, Pacchiarotti I, Lionetto L, De Rossi P, Sanna L, et al. Serum levels of risperidone and its metabolite, 9-hydroxyrisperidone: correlation between drug concentration and clinical response. Ther Drug Monit 2009;31: 475-481.

35. Ardic UA, Küçükköse M, Inci SB, Ercan ES. Efficacy and safety profile of risperidone long-acting injection in adolescents in a real-life setting. Clin Psychopharmacol Neurosci 2018;16: 57-61.

36. Demirkaya SK, Aksu H, Özgür BG. A retrospective study of long acting risperidone use to support treatment adherence in youth with conduct disorder. Clin Psychopharmacol Neurosci 2017; 15:328-336.

37. Juárez-Treviño M, Esquivel AC, Isida LML, Delgado DÁG, de la O Cavazos ME, Ocañas LG, et al. Clozapine in the treatment of aggression in conduct disorder in children and adolescents: a randomized, double-blind, controlled trial. Clin Psychopharmacol Neurosci 2019;17:43-53. 